Colloids and Surfaces A: Physicochemical and Engineering Aspects

MANUSCRIPT ID: COLSUA-D-16-00391

ISSN: 0927-7757 IMPACT FACTOR $=2.752$

Accepted June $8^{\text {th }} 2016$

\title{
TRANSVERSE MAGNETIC FIELD DRIVEN MODIFICATION IN UNSTEADY PERISTALTIC TRANSPORT WITH ELECTRICAL DOUBLE LAYER EFFECTS
}

\author{
${ }^{* 1}$ D. Tripathi, ${ }^{1}$ S. Bhushan and ${ }^{2}$ O. Anwar Bég \\ ${ }^{I}$ Department of Mechanical Engineering, Manipal University, Jaipur-303007, India. \\ ${ }^{2}$ Fluid Mechanics, Spray Research Group, Petroleum and Gas Engineering, Salford University, \\ Newton Building, G77, The Crescent, Salford, M54WT, England, UK.
}

*Corresponding author-email: dharmtri@gmail.com

\begin{abstract}
The influence of transverse magnetic field on time-dependent peristaltic transport of electrically-conducting fluids through a microchannel under an applied external electric field with induced electric field effect is considered, based on lubrication theory approximations. The electrohydrodynamic (EHD) problem is also simplified under the Debye linearization. Closed-form solutions for the linearized dimensionless boundary value problem are derived. With increasing Hartmann number, the formation of bolus in the regime (associated with trapping) is inhibited up to a critical value of magnetic field. Flow rate, axial velocity and local wall shear stress are strongly decreased with greater Hartmann number whereas pressure difference is enhanced with higher Hartmann number at low time values but reduced with greater elapse in time. With greater electro-osmotic parameter (i.e. smaller Debye length), maximum time-averaged flow rate is enhanced, whereas the axial velocity is reduced. An increase in electrical field parameter (i.e. maximum electro-osmotic velocity) causes an increase in maximum time-averaged flow rate. The simulations find applications in electromagnetic peristaltic micro-pumps in medical engineering and also "smart" fluid pumping systems in nuclear and aerospace industries.
\end{abstract}

Keywords: Electric field; Debye length; Magnetic field; Hartmann number; Trapping; Medical and smart micro-pumps. 


\section{INTRODUCTION}

Significant technological advances have been made in recent years in the field of microscale pumping designs including the development of microfluidics devices, micropumping and peristaltic micropumping [1]. An electro-osmotic pump is capable of generating high pressure and flow without moving mechanical parts and a robust design in this regard has been presented by Goodson et al. at the Nano Heat Mechanical Engineering Laboratory, Stanford University, USA [2]. Peristalsis is a physiological mechanism which achieves excellent and efficient transport of materials via periodic contraction of the conveying conduit. In this sense it is a smart system as it responds to electrical nerve stimulation and is mobilized intelligently to varying needs. Exploiting this principle of peristalsis, an electrostatically-actuated, biomimetic, micro- peristaltic pump has also been designed and fabricated by Xie et al. [3]. A microfluidic peristaltic pump has further been developed by Thomas and Dorrer [4]. Most recently, a microfluidic device comprising pumps, valves, and fluid oscillation dampers has been engineered by Chou et al. [5]. Furthermore an efficient, valve-less microfluidic peristaltic pumping method has been introduced by Zhang et al. [6]. A theoretical study on electromagneto-hydrodynamic micropumps using Jeffrey viscoelastic working fluids in two parallel microchannels has been reported by Si and Jian [7]. As elaborated earlier, peristalsis is a unique mechanism in biology which comprises an automatic periodic series of muscle contractions and relaxation that occurs during movement of, for example, food bolus through the digestive system, urine flow from the kidneys into the bladder, transportation of bile from the gall bladder into the duodenum and many other physiological systems. Peristalsis has been studied in medical sciences for over a century. In the context of theoretical and experimental fluid dynamics, it received attention in the mid-1960s onwards, again motivated by the development of efficient pumps for medical and other applications. Important mathematical works in this regard have been communicated by Burns and Parkes [8], Fung and Yih [9], Shapiro et al. [10], Jaffrin and Shapiro [11], Shukla et al. [12], Takabatake, and Ayukawa [13], Pozrikidis [14] and Li and Brasseur [15]. More recent studies which have extended the purely Newtonian fluid models described in [8]-[15] include Tripathi and Bég [16] who examined viscoelastic peristaltic propulsion, Tripathi and Bég [17] who studied peristaltic flows of magnetized couple stress fluids, Blanchette [18] who considered the influence of suspended drops on peristaltic pumping, Ellahi et al. [19] who computed heat transfer in peristaltic flow. Further recent studies exploring other areas of peristaltic transport are the articles of Tripathi and Bég [20] concerning 
nanofluids, Kothandapani and Prakash [21] on magnetic nanofluids and Akbar et al. [22] also on magnetic nanofluids in permeable channels.

Although, as described earlier, numerous experimental studies have been communicated concerning electrohydrodynamic (EHD) and magnetohydrodynamic (MHD) peristaltic micropumps, relatively few investigations of a theoretical nature in the former have been presented. Mathematical and computational models provide nowadays an essential compliment to experimental studies. They further allow the optimization of new designs which are critical for sustained performance in many areas including medicine, process, aerospace and nuclear engineering. Although Bég et al. [23] studied electrohydrodynamic flows in diabetic micropumps and included electrical Reynolds number and electrical slip effects, they did not consider the peristaltic mechanism i.e. the analysis in [23] was confined to a non-deformable channel. Electrokinetic theory was first applied to peristaltic transport by Chakraborty [24] who explored the deployment of an axial electric field (and therefore an axial electro-kinetic body force) for enhancing microfluidic pumping rates in peristaltic microtubes and examined the modes of interaction between the electro-osmotic and peristaltic mechanisms for the effects of occlusion number, characteristic electro-osmotic velocity and the peristaltic wave speed. This work demonstrated that for small occlusion numbers, an axial electrokinetic body force in peristalsis may successfully augment the time-averaged flow rate and improve efficiency considerably. The work in [24] however was confined to the case of an electroosmotic slip boundary condition and external electric field effects were neglected in the model. Electrokinetics is the study of a group of numerous complex phenomena which occur in particles (solid, liquid or gas) containing fluids which respond to an intrinsic or extrinsic electric field. Essentially it involves the hydrodynamics of the movement of ionic solutions in the vicinity of electrically-charged interfaces. The seminal work in this field is that of Saville [25], which addresses both chemical engineering and biophysical phenomena. Electrokinetic phenomena include di-electrophoresis (the force applied to polar liquids in the presence of nonuniform electric fields which has also been utilized for digital bio-microfluidics), electroosmosis, diffusiophoresis, capillary osmosis, sedimentation potential, streaming potential/current, colloid vibration current and electric sonic amplitude effects, to name a few. Attracted by ever-growing applications of electrokinetics in various fields (including nanotechnology), a number of investigators have more recently studied the electrokinetic flows in various configurations. Vakili et al. [26] investigated the hydrodynamically fully developed electro-osmotic flow of power-law fluids in rectangular microchannels, solving the Poisson 
electrical potential and momentum equations with a computational finite difference procedure utilizing a non-uniform grid. They showed that dimensionless mean velocity (and electrical zeta potential) is elevated with channel aspect ratio and the dimensionless Debye-Hückel parameter but depressed with rheological power law index. Chakraborty and Paul [27] analyzed the collective effects of electrical and magnetic forces in micro-channel flow control. They simulated the electric double layer (EDL) effects using the classical Poisson-Boltzmann equation, and found that volumetric flow rates are significantly increased with comparatively weak magnetic field, whereas with stronger magnetic fields, significant volumetric forces can oppose and inhibit flow rate augmentation. Other studies include Dey et al. [28] who examined heat transfer in electro-osmotic and pressure-driven flows in narrow confinements with thick electric double layers [28]. Mohammadi et al. [29] reported very recently on hydrodynamic and direct-current insulator-based dielectrophoresis (H-DC-iDEP) microfluidic blood plasma separation. Sheikholeslami and Ellahi [30] studied transport in electrohydrodynamic nanofluids with sinusoidal upper wall and hydrothermal effects.

However the models described in [25]-[30] ignored the peristaltic effect. Recently however several authors have considered electro-kinetic peristaltic transport, extending the earlier work of Chakraborty [24]. El-Sayed et al. [31] studied the influence of an alternating vertical electric field and heat transfer on a peristaltic flow of an incompressible dielectric viscoelastic (Oldroyd) fluid in a symmetric flexible channel. They derived perturbation solutions for electric field, flow field and temperature distributions, and noted that backflow may be induced close to the lower bound of the channel with greater electrical Rayleigh number, Reynolds number and Weissenberg (viscoelastic) numbers. They further observed that trapped bolus magnitude is reduced at the upper bound of the channel and increases at the lower bound of the channel with higher electrical Rayleigh number, with the contrary behaviour for increasing temperature parameter. Very recently Goswami et al. [32] investigated theoretically the electro-kinetically modulated peristaltic transport of power law fluids through a narrow deformable tube delineated into two regions (a non-Newtonian core region (described by the power-law behavior) engulfed by a thin wall-adhering layer of Newtonian fluid), aimed at simulating the wall-adjacent cell-free skimming layer in blood samples typically handled in microfluidic transport. Their computations showed that the influence of electro-osmosis on pressure rise is prevalent at lower occlusion values (which highlights the significance for transport modulation in weakly peristaltic flow) and that trapping is successfully regulated via 
electric field and infect is eliminated at sufficiently strong electrical field strengths as is the reflux phenomenon.

Motivated from the combined applications of peristalsis and electrokinetic transport in fabrication of microfluidic pumping devices, a new fluid mechanical model is developed in the present work, to discuss the effects of external electric field and thickness of electric double layer (EDL) on peristaltic pumping of viscous fluids through microchannels. A non-integral number of fluid boluses are taken as propagating along the microchannel length. The potential for electrokinetic peristaltic pumps may be considerable [33-34]. Electrokinetic pumping without peristalsis has been shown to be limited to relatively few applications e.g. capillary electrophoresis which is ideal for electroosmotic) but presents a number of difficulties including the micro-machining expense of specific surfaces needed to operate (usually glass), over-sensitivity to ionic composition of the buffer (hydroxyl group dissociation at the walls), relatively high-voltage sources and switches which are costly and difficult to install and also may have associated health hazards. Many of these drawbacks are considered in [35]-[41]. Other works have rigorously explored magnetohydrodynamic peristaltic pumping and identified certain advantages and disadvantages of this method. These include the studies of Kumari and Radhakrishnamacharya [42] and Ramesh and Devakar [43] which have also incoporated slip effects and couple stress rheological effects. Other researchers have explored magnetohydrodynamic transport with a range of different formulations including Lorentz body forces [44], streamwise magnetic field [45], ferrohydrodynamics [46], magnetic nanofluids [47], magnetic induction [48] and mesoscopic hydromagnetic heat transfer [49]. Furthermore magnetohydrodynamic micropumps (which propel conductive liquids which are subjected to perpendicular applied electric and magnetic fields across a microchannel via the Lorentz force) suffer from bubble problems associated with electrolysis which can seriously inhibit flow and reduce efficiency and the range of applications, especially in medicine. Therefore a principal objective of the present study is to combine magnetohydrodynamic and electro-kinetic phenomena to explore the resulting performance in peristaltic transport mechanisms. Currently there is no universally recognized standard for achieving the optimized performance of micropumps. Different designs are dependent on many factors including operating mechanism, construction materials, expense, portability, relative facility for fabrication, bio-compatibility and re-usability. The present work therefore investigates the fluid mechanics of magnetized peristaltic pumping with electrokinetic transport. Integral to this is the need to establish exactly how peristaltic pumping can be controlled by Debye length thickness and strength of applied 
external electric field. The Debye length quantifies the charge carrier's net electrostatic effect in solution, and to what physical extent such electrostatic effects persist. It has different meaning in plasma physics, semi-conductor materials and electro-kinetics. We elaborate at length the influence of this parameter in addition to Hartmann (magnetic body force) parameter. Extensive visualization of flows is also included as are the effects of time and other parameters on pressure and velocity distributions in a finite channel as this geometry is physically more realistic for actual engineered systems. Detailed interpretations of the flow phenomena are presented. The present mathematical model is therefore envisaged to be of some significance in the field of biomimetic microfluidics utilizing peristalsis and electromagnetic flow phenomena in the pumping process.

\section{MATHEMATICAL FORMULATION}

The geometric model for the electrokinetic peristaltic transport under transverse magnetic field through a finite length $(L)$ channel, as a simulation of an electromagnetic biomimetic pump, depicted in Fig.1, is mathematically defined according to the following relations:

$$
\bar{h}(\bar{\xi}, \bar{t})=\left\{\begin{array}{l}
a-\bar{\phi} \cos ^{2} \frac{\pi}{\lambda}(\bar{\xi}-c \bar{t}), \text { if } t<\xi<t+1 \\
a-\bar{\phi}, \quad \text { if } \xi \in[0, t] \cup[t+1, L]
\end{array},\right.
$$

where $a, \bar{\phi}, \lambda, \bar{\xi}, c, \bar{t}, L$ are the radius of tube, amplitude, wavelength, axial coordinate, wave velocity, time and channel length. 


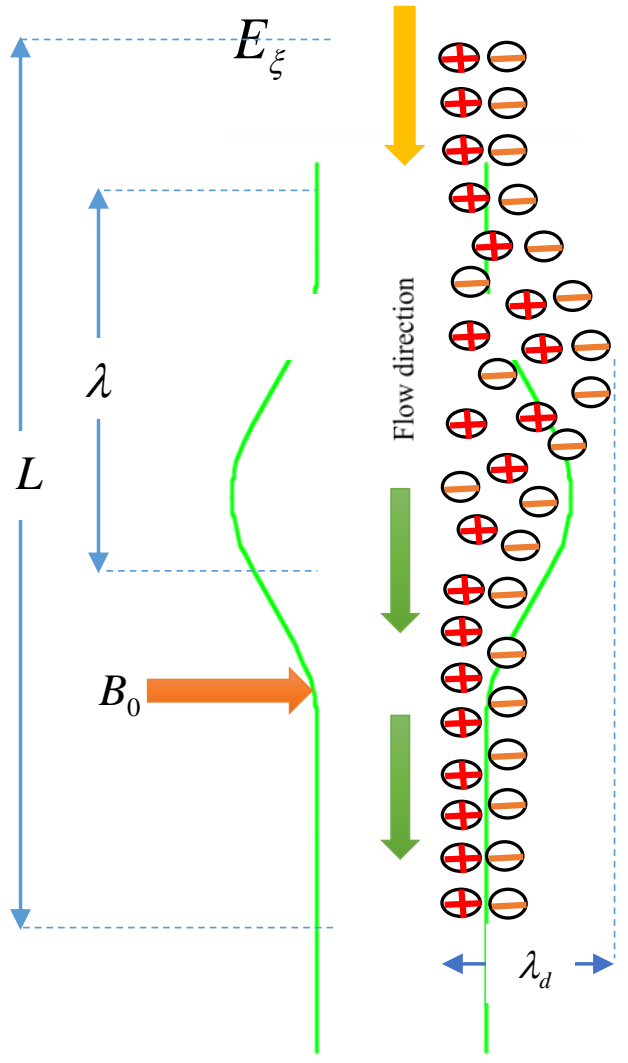

Fig.1. Geometry of conductive peristaltic pumping with electrical and magnetic field effects.

The governing equations for unsteady, two-dimensional, electrically-conducting, incompressible flow with an applied electrokinetic body force in the axial (longitudinal) direction and static magnetic field in the transverse direction (i.e. mutually orthogonal subject to Maxwell's equations) can be shown to take the form:

$$
\begin{aligned}
& \vec{\nabla} \cdot \vec{V}=0, \\
& \rho\left\{\frac{\partial \vec{V}}{\partial t}+(\vec{V} \cdot \vec{\nabla}) \vec{V}\right\}=-\vec{\nabla} p+\mu \vec{\nabla}^{2} \vec{V}+\bar{\rho}_{e} \vec{E}+\vec{J} \times \vec{B} .
\end{aligned}
$$

where $\rho, \vec{V}, \bar{p}, \mu, \vec{E}$, and $\bar{\rho}_{e}$ denote the fluid density, velocity vector, pressure, fluid viscosity, electrokinetic body force, and the density of the total ionic change respectively and $\vec{J}=\sigma(\vec{E}+\vec{V} \times \vec{B})$ is the electric current density (based on Ohm's law), $\vec{B}$ is magnetic field, $\sigma$ is the electrical conductivity of the fluid. The Poisson-Boltzmann equation for electric potential distribution (developed due to the presence of the electrical Debye layer i.e. EDL) is described as: 


$$
\nabla^{2} \bar{\Phi}=-\frac{\bar{\rho}_{e}}{\varepsilon}
$$

where $\varepsilon$ is the permittivity and $\rho_{e}=e z\left(n^{+}-n^{-}\right)$, in which $n^{+}$and $n^{-}$are the number of densities of cations and anions respectively and are given by the Boltzmann distribution (considering no EDL overlap), viz, $n^{ \pm}=n_{0} \operatorname{Exp}\left[ \pm \frac{e z \bar{\Phi}}{K_{B} T}\right]$, where $n_{0}$ represents the concentration of ions at the bulk, which is independent of surface electro-chemistry, $e$ is the electronic charge, $z$ is charge balance, $K_{B}$ is the Boltzmann constant, $T$ is the average temperature of the electrolytic solution. Introducing a normalized electro-osmotic potential function $\bar{\Phi}$ with zeta potential $\zeta$ of the medium along with other non-dimensional variables, like $\Phi=\frac{\bar{\Phi}}{\zeta} \quad$ and applying Debye-Huckel linearization approximation, namely $\sinh \left(\frac{e z \Phi}{K_{B} T}\right) \approx \frac{e z \Phi}{K_{B} T}$, and imposing the boundary conditions $\left.\frac{\partial \Phi}{\partial \eta}\right|_{\eta=0}=0$ and $\left.\Phi\right|_{\eta=h}=1$, the potential function is obtained as:

$$
\Phi=\frac{\cosh (m \eta)}{\cosh (m h)}
$$

where $m=a e z \sqrt{\frac{2 n_{0}}{\varepsilon K_{B} T}}=\frac{a}{\lambda_{d}}$, is known as the electro-osmotic parameter and $\lambda_{d} \propto \frac{1}{m}$ is Debye length or characteristic thickness of electrical double layer (EDL). Under the assumptions of long wave length and low Reynolds number, the electro-magnetohydrodynamic peristaltic flow is governed by the following equations:

$$
\begin{aligned}
& \frac{\partial u}{\partial \xi}+\frac{\partial v}{\partial \eta}=0 \\
& \frac{\partial p}{\partial \xi}=\frac{\partial^{2} u}{\partial \eta^{2}}-H a^{2} u+m^{2} U_{H S} \Phi
\end{aligned}
$$

where $u, v, \xi, \eta$ are the axial velocity, transverse velocity, axial coordinate and transverse coordinate respectively, $\operatorname{Re}=\frac{\rho c a}{\mu}$, is the Reynolds number, $H a=B_{0} a \sqrt{\frac{\sigma}{\mu}}$, is the Hartmann (magnetohydrodynamic body force) number, $U_{H S}=-\frac{E_{\xi} \varepsilon \zeta}{\mu c}$ is the Helmholtz-Smoluchowski 
velocity or maximum electro-osmotic velocity and $E_{\xi}$ is axially-applied electric field. The imposed boundary conditions are:

$$
\left.\frac{\partial u}{\partial \eta}\right|_{\eta=0}=0,\left.u\right|_{\eta=h}=0,\left.v\right|_{\eta=0}=0,\left.v\right|_{\eta=h}=\frac{\partial h}{\partial t},\left.p\right|_{\xi=0}=p_{0} \text { and }\left.p\right|_{\xi=L}=p_{L}
$$

Integrating Eq. (7) and using boundary conditions (8), the axial velocity is obtained as:

$$
u=\frac{1}{H a^{2}} \frac{\partial p}{\partial \xi}\left\{\frac{\cosh (H a \eta)}{\cosh (H a h)}-1\right\}+\frac{m^{2} U_{H S}}{m^{2}-H a^{2}}\left\{\frac{\cosh (H a \eta)}{\cosh (H a h)}-\frac{\cosh (m \eta)}{\cosh (m h)}\right\}
$$

Using Eqn. (9) and boundary condition (8), the transverse velocity from the continuity equation (6) is obtained as:

$$
\begin{aligned}
v=- & \frac{1}{H a^{2}}\left\{\frac{\partial^{2} p}{\partial \xi^{2}}\left(\frac{\sinh (H a \eta)}{H a \cosh (H a h)}-\eta\right)+\frac{\partial p}{\partial \xi} \frac{\partial h}{\partial \xi} \frac{\sinh (H a \eta) \tanh (H a h)}{\cosh (H a h)}\right\}+ \\
& \frac{m^{2} U_{H S}}{m^{2}-H a^{2}}\left\{\frac{\sinh (H a \eta) \tanh (H a h)}{\cosh (H a h)}-\frac{\sinh (m \eta) \tanh (m h)}{\cosh (m h)}\right\} \frac{\partial h}{\partial \xi}
\end{aligned}
$$

Furthermore, utilizing Eqn. (10) and boundary condition (8), the pressure gradient emerges as:

$$
\frac{\partial p}{\partial \xi}=\frac{H a^{3}}{H a h-\tanh (H a h)}\left[G_{0}(t)+\int \frac{\partial h}{\partial t} d \xi-\frac{m^{2} U_{H S}}{m^{2}-H a^{2}}\left\{\frac{\tanh (H a h)}{H a}-\frac{\tanh (m h)}{m}\right\}\right]
$$

where $G_{0}(t)$ is arbitrary function of time $(t)$ to be evaluated by using finite length boundary conditions. The pressure difference can be computed along the axial length by the expression:

$$
\Delta p=p(\xi, t)-p(0, t)=\int_{0}^{\xi} \frac{\partial p}{\partial s} d s
$$

Here $G_{0}(t)$ is expressed as:

$$
G_{0}(t)=\frac{\left(p_{l}-p_{0}\right)-\int_{0}^{L} \frac{H a^{3}}{H a h-\tanh (H a h)}\left[\int \frac{\partial h}{\partial t} d \xi-\frac{m^{2} U_{H S}}{m^{2}-H a^{2}}\left\{\frac{\tanh (H a h)}{H a}-\frac{\tanh (m h)}{m}\right\}\right] d \xi}{\int_{0}^{L} \frac{H a^{3}}{H a h-\tanh (H a h)} d \xi} .
$$

The local wall shear stress is defined as: 


$$
\tau_{w}=\left.\frac{\partial u}{\partial \eta}\right|_{\eta=h}=\frac{\tanh (H a h)}{H a} \frac{\partial p}{\partial \xi}-\frac{m^{2} U_{H S}}{m^{2}-H a^{2}}\{H a \tanh (H a h)-m \tanh (m h)\} .
$$

The flow rate is defined as:

$$
Q(\xi, t)=\int_{0}^{h} u d \eta=\frac{1}{H a^{3}} \frac{\partial p}{\partial \xi}\{\tanh (H a h)-H a h\}+\frac{m^{2} U_{H S}}{m^{2}-H a^{2}}\left\{\frac{\tanh (H a h)}{H a}-\frac{\tanh (m h)}{m}\right\}
$$

The time averaged volume flow rate may then be defined as:

$$
\bar{Q}=\int_{0}^{1} Q d t=Q-h+1-\phi / 2
$$

Using Eqn. (9), the stream function in the wave frame (obeying the Cauchy-Riemann equations, $u=\frac{\partial \psi}{\partial \eta}$ and $v=-\frac{\partial \psi}{\partial \xi}$ ) takes the form:

$$
\psi=\frac{1}{H a^{2}} \frac{\partial p}{\partial \xi}\left\{\frac{\sinh (H a \eta)}{H a \cosh (H a h)}-\eta\right\}+\frac{m^{2} U_{H S}}{m^{2}-H a^{2}}\left\{\frac{\sinh (H a \eta)}{H a \cosh (H a h)}-\frac{\sinh (m \eta)}{m \cosh (m h)}\right\} .
$$

All the above expressions will reduce to the corresponding expressions for peristaltic transport of viscous fluids through finite length channel with $U_{H S}=0$ i.e. for vanishing HelmholtzSmoluchowski velocity (maximum electro-osmotic velocity). It is also noteworthy that the expressions (9)-(17) contract to the case for electro-kinetic peristaltic transport through a very thin electric double layer with electro-osmotic parameter $m \rightarrow \infty$. Finally for $H a \rightarrow 0$, magnetic body force effects are negated and the general model reduces to purely electro-kinetic peristaltic transport without magnetic field.

\section{RESULTS AND DISCUSSION}

A parametric study of the influence of the key electro-magnetic and hydrodynamic parameters on the flow variables has been conducted. Solutions are illustrated in Figs. 2-6. 

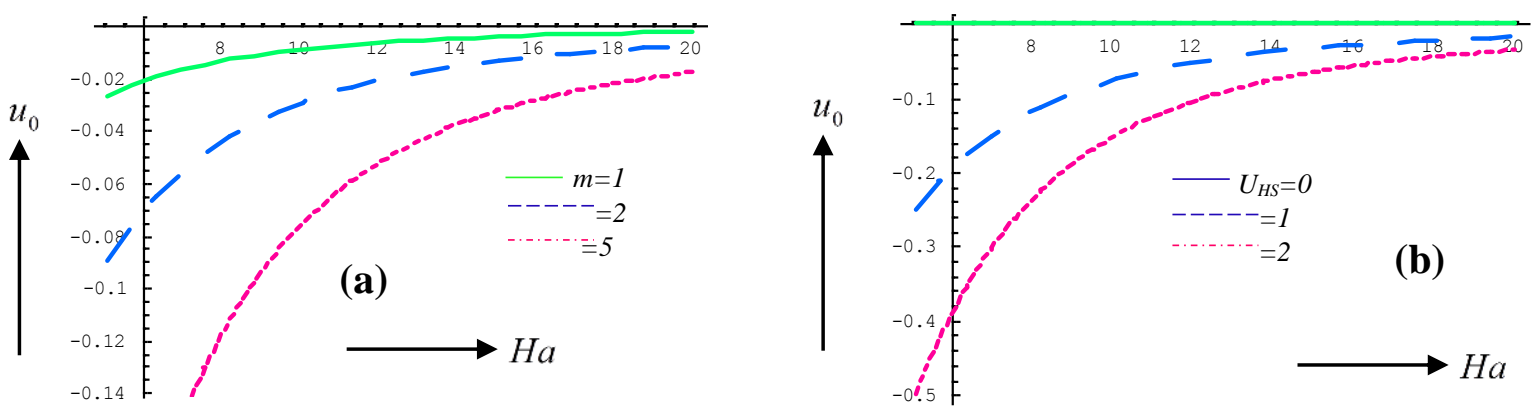

Fig.2. Initial axial velocity profile vs. Hartmann number at $\varphi=0.6$, and (a) $\mathrm{U}_{H s}=1$ for different Debye length (b) $m=5$ for different external electric field

(b)

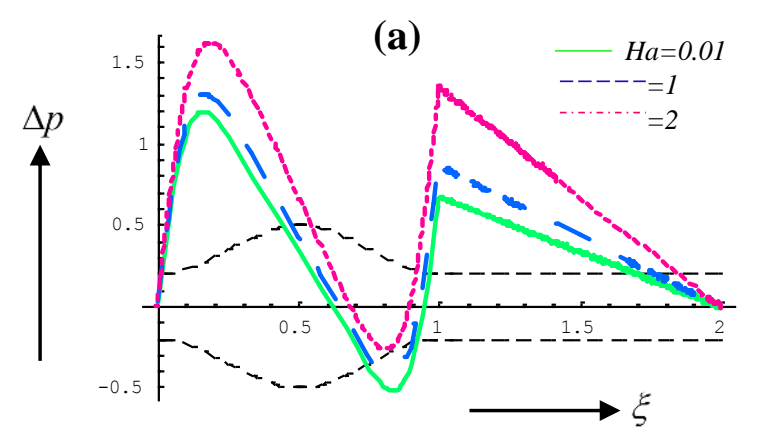

(c)

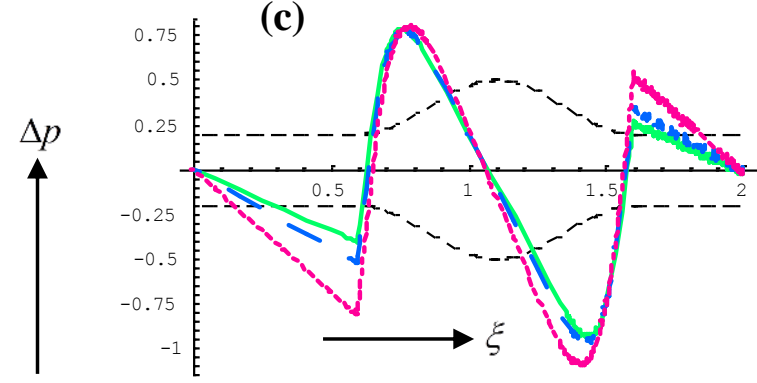

(b)

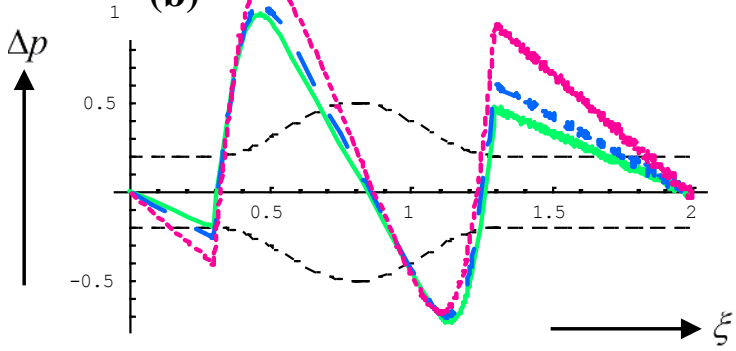

(d)

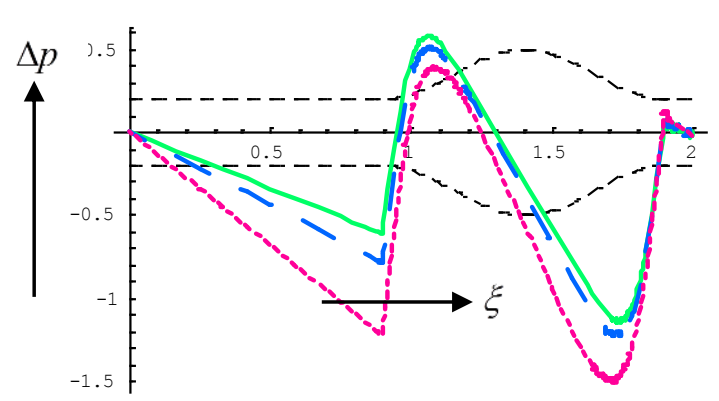

Fig.3. Pressure distribution along the length of channel at $\varphi=0.6, l=2, p_{l}=p_{0}=0, U_{H S}=1, m=5$. Color lines represent the pressure distribution for different values of Hartmann number at (a) $t=0$, (b) $t=0.3$, (c) $t=0.6$, (d) $t=0.9$. Black lines show the single wave propagation. 

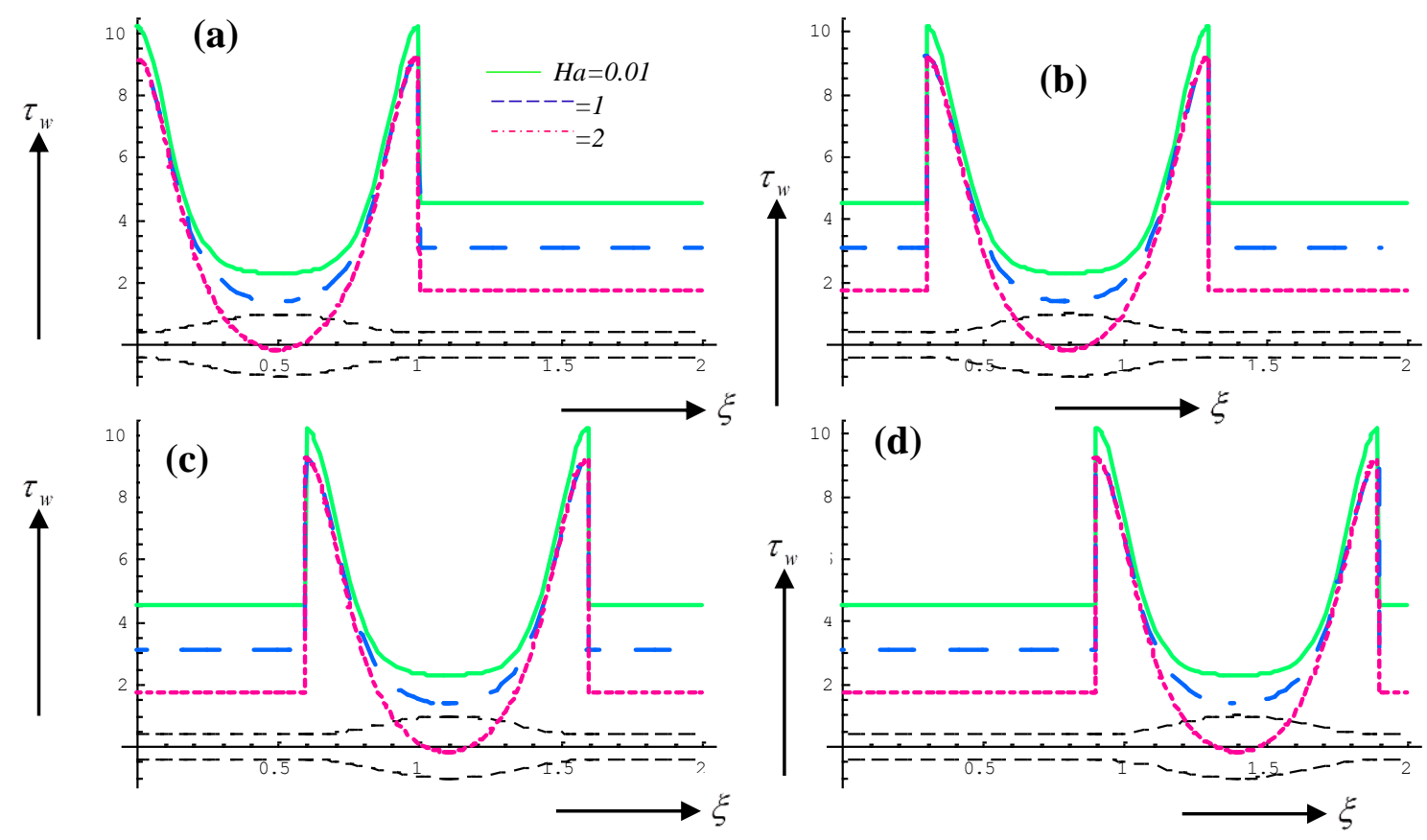

Fig.4. Local wall shear stress along the length of cnannel for $\varphi=0.6, l=2, p_{l}=p_{0}=0, U_{H S}=1, m=5$. Color lines represent the Local wall shear stress for different values of Hartmann number at (a) $t=0$, (b) $t=0.3$, (c) $t=0.6$, (d) $t=0.9$. Black lines show the single wave propagation.

(a)

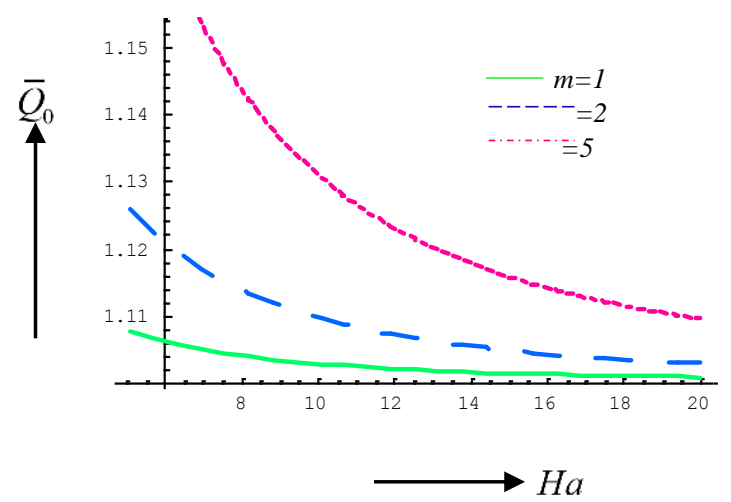

(b)

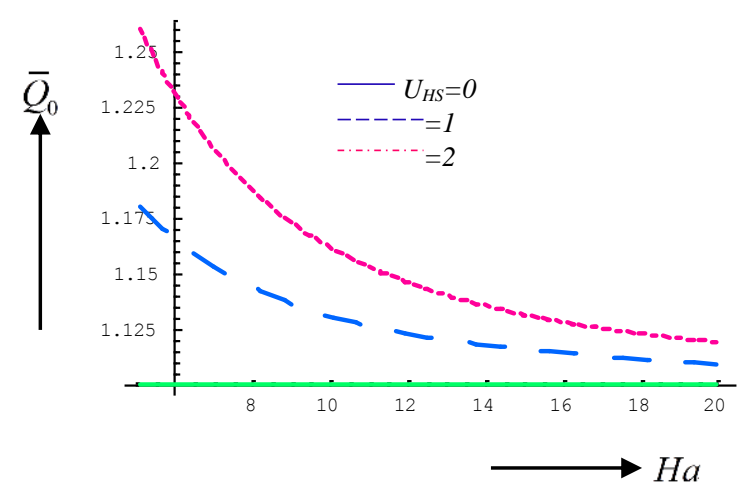

Fig.5. Maximum time averaged flow rate flow vs. Hartmann number at $\varphi=0.5$, and (a) $\mathrm{U}_{H s}=1$ for different Debye length (b) $m=5$ for different external electric field. 

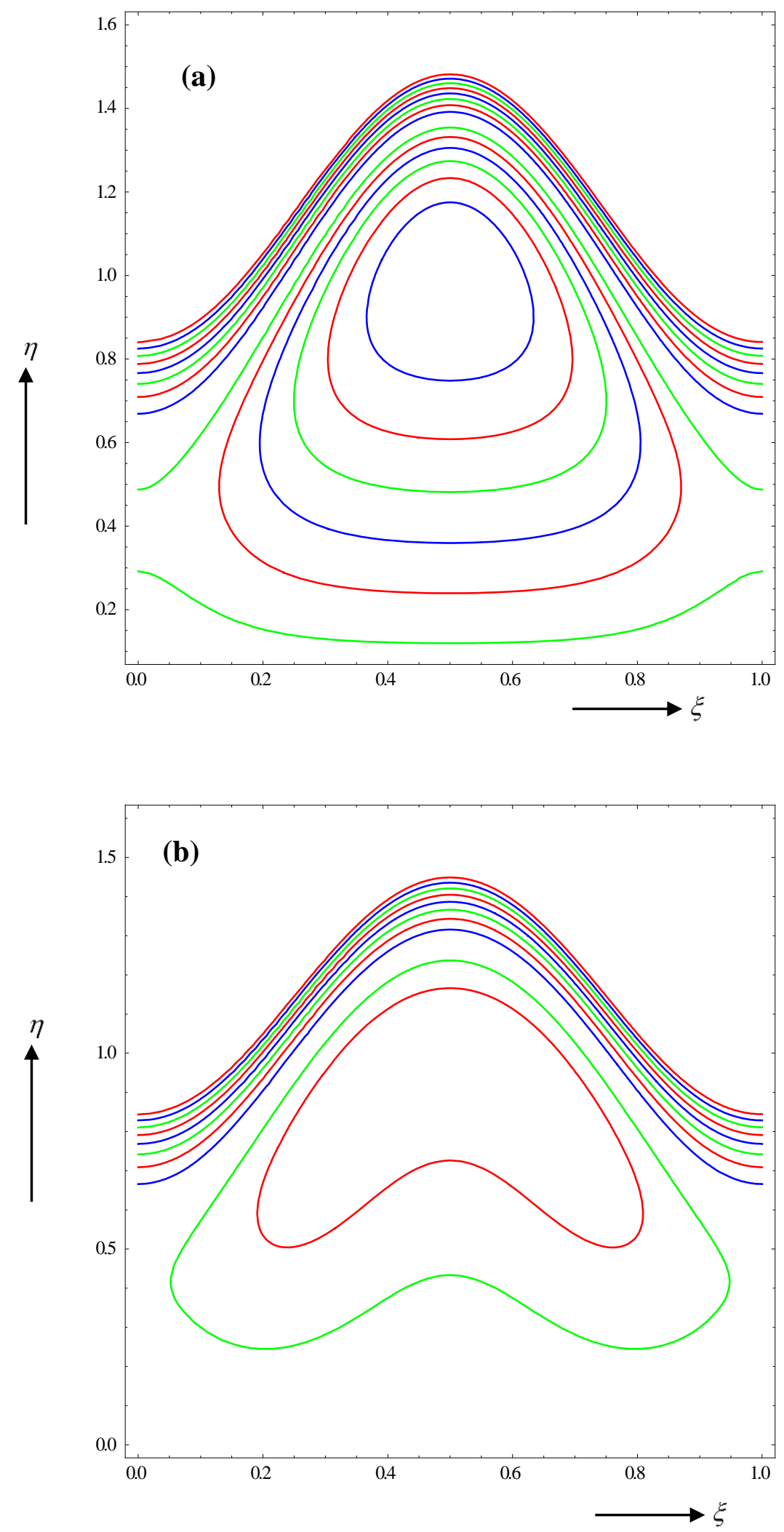

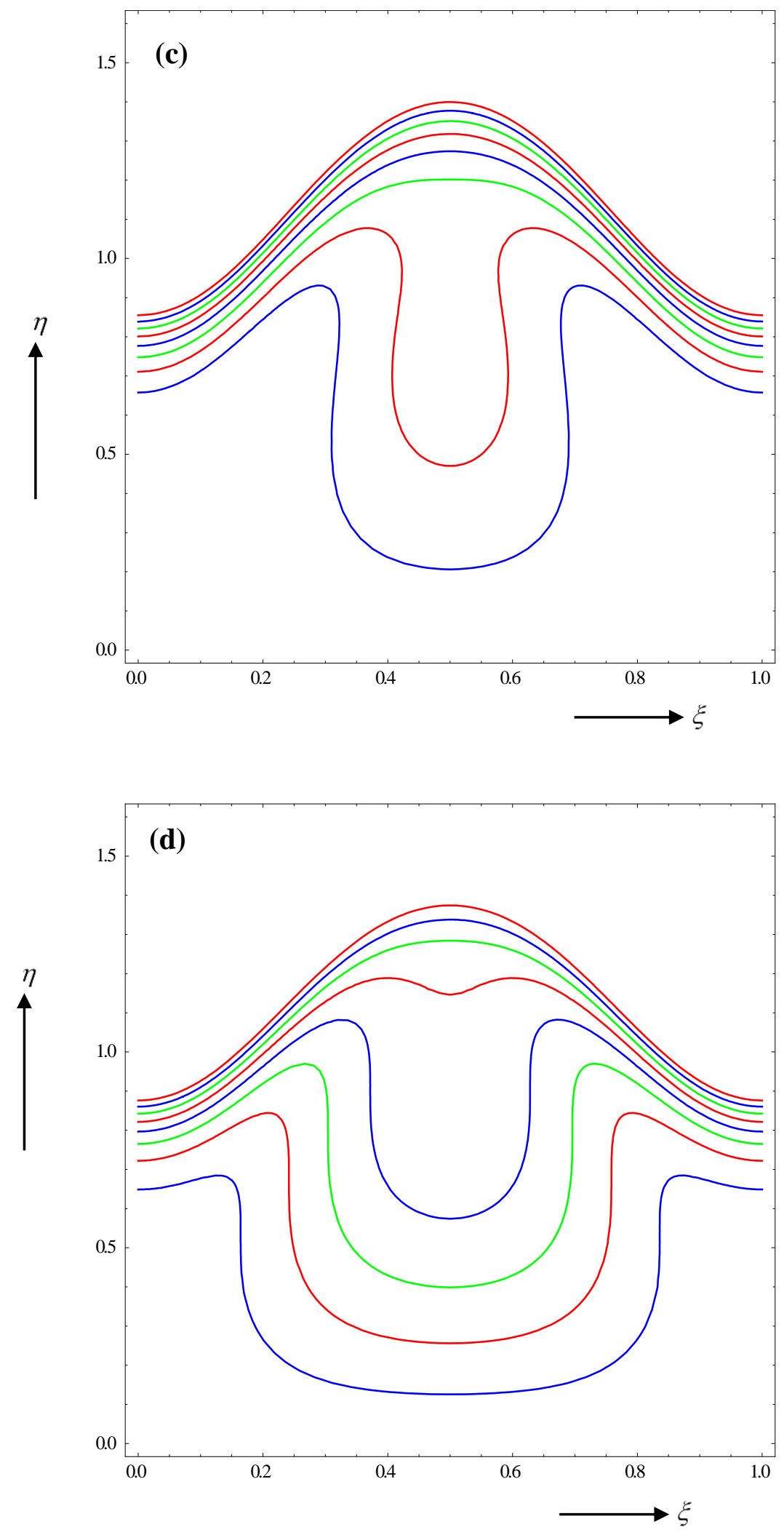

Fig.6. Stream lines at $\varphi=0.6, \bar{Q}=0.6, U_{H S}=1, m=5$ (a) $H a=0.001$, (b) $H a=1$,

(c) $H a=2$, (d) $H a=3$ 
Figs. 2a-b depict the variation in initial axial velocity $\left(u_{o}\right)$ computed against Hartmann number ( $\mathrm{Ha}$ ) for respectively: a) electro-osmotic parameter $(\mathrm{m})$ which itself is inversely proportional to Debye length $\left(m=a / \lambda_{d}\right)$ and b) $U_{H S}$ i.e. maximum electro-osmotic velocity (which follows a direct proportionality with external applied electrical field, $E_{\xi}$, since $\left.U_{H S}=-\frac{E_{\xi} \varepsilon \zeta}{\mu c}\right)$. Axial velocity is significantly increased with greater Hartmann number, as testified to by the decrease in negative values of $u_{o}$ with higher $H a$. Since $H a=B_{0} a \sqrt{\frac{\sigma}{\mu}}$, which defines the relative influence of magnetic body force to viscous hydrodynamic force, therefore the implication is that greater transverse magnetic field $\left(B_{0}\right)$ and therefore Lorentzian body force $\left(-H a^{2} u\right.$ in eqn.(7)) induces a strong acceleration in the axial flow. Conversely with increasing electroosmotic parameter, $m$, (i.e. smaller Debye length) there is a substantial deceleration in the axial flow i.e. the axial velocity values become increasingly negative. The electrostatic body force, $m^{2} U_{H S} \Phi$, therefore inhibits flow in this regime whereas the magnetic body force has the opposite influence. It is also worth emphasizing that the magnitudes of velocity in fig. $2 \mathrm{a}$ are evidently an order of magnitude greater than those in fig. 2a. However, effectively the electrokinetic effect (linked to electrostatic axial body force) achieves better hydrodynamic control than the magneto-hydrodynamic body force and this is of great relevance to precision design in micro-pumps $[34,35]$.

Figs. 3a-d present the evolution in pressure difference $(\Delta p)$ with axial coordinate $(\xi)$, time $(t)$ and also for various Hartmann numbers $(\mathrm{Ha})$. The peristaltic wave propagation profile is visualized in black dotted lines and clearly corresponds to a single wave. It is evident that the pressure distribution does not follow the predictable sinusoidal variation (characteristic of conventional peristaltic fluid mechanics) and that some degree of damping is introduced, probably owing to the interaction of electro-kinetic and magnetic body force. Smooth and sharp peaks and troughs are found to arise in an alternating fashion. The periodic nature of the flow is however clearly captured which is associated with peristaltic wave dynamics owing to the deformable channel walls. With increasing Hartmann number there is a significant elevation in the pressure difference (figs $3 \mathrm{a}, \mathrm{b}$ ) at $t=0$ (initiation of flow and $t=0.3$, respectively. However with further progression in time the reverse effect is computed i.e. pressure differences are suppressed with greater Hartmann number. Therefore with regard to real-time electro-magnetic micro-pumps, Hartmann number while initially achieving a boost in hydrodynamic pressure, 
with further time elapse causes the contrary effect. Pressure differences are also found to be reduced with greater axial distance $(\xi)$, i.e. further from the inlet of the channel.

Figs. 4a-d illustrate the profiles for local wall shear stress $\left(\tau_{w}\right)$ again with axial coordinate $(\xi)$, time $(t)$ and Hartmann number $(\mathrm{Ha})$. Again the peristaltic wave propagation profile is visualized in black dotted lines and a single wave is captured. At $t=0$, as shown in figure $4 \mathrm{a}$, following an initial decay in wall shear stress with small change in axial coordinate, there ensues an equally rapid ascent and after this a very steep plummet (almost vertical drop) at intermediate distance from the channel inlet. Thereafter a step change in wall shear stress is observed for greater subsequent values of the axial coordinate. A peak in wall shear stress therefore only arises once at a single location from the inlet. However with subsequent elapse in time (figs $4 \mathrm{~b}, \mathrm{c}, \mathrm{d}$ ) a dual peak is witnessed with a double step change. The initial peak is further displaced from the inlet with increasing time. Evidently the time-dependent nature of the peristaltic wave is reflected in the step-changes in wall stress and these have also been computed by other researchers including Smits [34]. With increasing Hartmann number the wall shear stress is invariably suppressed and at least once in each plot momentarily becomes negative implying flow reversal.

Figs 5a,b depict the distribution in maximum averaged volumetric flow rate $\left(\bar{Q}_{0}\right)$ with different Hartmann number $(\mathrm{Ha})$ and also Debye length parameter $(m)$ and external electrical field parameter $\left(U_{H S}\right)$. Flow rate (fig. 5a) is clearly significantly reduced with greater Hartmann number i.e. a decrease in fluid transported per unit time is caused by greater magnetic field, which concurs with the decrease in wall shear stress computed earlier (fig. 4a, b). The converse response is sustained with greater electro-osmotic parameter (smaller Debye length) i.e. electro-kinetic effect and evidently a marked elevation in flow rate is observed at any Hartmann number. Similarly in fig. $5 \mathrm{~b}$ an increase in electrical field parameter (i.e. maximum electroosmotic velocity), $\left(U_{H S}\right)$ is also found to substantially enhance maximum time-averaged flow rates. The magnitudes of flow rates achieved in both figs $5 \mathrm{a}, \mathrm{b}$ are similar. The graphs confirm experimental findings e.g. [32] that axial electrical field and electro-kinetic effects are beneficial to the peristaltic pumping process and may be exploited to this effect in micro-pump designs.

Finally figs 6a-d illustrate the streamline distributions with Hartmann number. In all cases strong electrical field effects are present $\left(U_{H S}=1, m=5\right)$. This visualization allows a better examination of the so-called trapping phenomenon, wherein an internally circulating bolus of the fluid is formed by closed streamlines. For $\mathrm{Ha} \ll<1(\mathrm{Ha}=0.001)$, i.e. fig. 6a, the magnetic 
body force is significantly weaker than viscous force. The dominant viscosity results in the manifestation of a dual system of distinct boluses encapsulated with other regions of trapped flow. The streamlines immediately adjacent and parallel to the $\xi=0$ line are largely undistorted and this line delineates the two bolus zones. When $H a=1$ (fig. 6b), the magnetic force is increased a thousand-fold (it is now of the same magnitude as the viscous force) and this serves to significantly distort the dual bolus zones. With further increase in Hartmann number to 2 (fig. 6c) the dual zones lose the strong concentration of streamlines surrounding each of them, and a partial bolus is observed in each zone. However with further increase in Hartmann number $(\mathrm{Ha}=3)$ in fig. $6 \mathrm{~d}$, there is a re-appearance of the streamlines around each bolus and the formation of distinct zones becomes intensified again. Evidently therefore a critical Hartmann number exists somewhere between $H a=2$ and 3 in which the initially assistive and stabilizing nature of magnetic field is replaced with a disturbing nature. This has also been noted by several researchers including

\section{CONCLUSIONS}

Analytical solutions have been derived for the unsteady peristaltic transport of Newtonian electrically-conducting fluid in a microchannel under mutually perpendicular applied external magnetic and electric fields, under low Reynolds number and long wavelength approximations. The electro-kinetics has been simplified via the Debye linearization. The study has been motivated by exploring the combined use of magnetohydrodynamics and electrokinetics in biomimetic micro-pumps for possible medical applications. The present computations have shown that:

- With increasing Hartmann number, the formation of bolus in the regime is inhibited up to a critical value of magnetic field. Flow rate is however reduced with greater Hartmann number as is the local wall shear stress and the magnitude of axial velocity. Pressure difference is also found to be increased with greater Hartmann number at low time values whereas it is reduced with greater elapse in time. Furthermore increasing Hartmann number decreases local wall shear stress values.

- With greater electro-osmotic parameter (i.e. smaller Debye length), maximum timeaveraged flow rate is enhanced, whereas there is a strong deceleration in the axial flow.

- With an increase in electrical field parameter (i.e. maximum electro-osmotic velocity), maximum time-averaged flow rate is significantly elevated.

- With greater axial distance, pressure differences are markedly decreased. 
The present work has ignored rheological working fluids. These will be addressed in the near future using different non-Newtonian approaches.

\section{ACKNOWLEDGMENTS}

The authors are grateful to all 3 reviewers for their comments which have served to improve the present article.

\section{REFERENCES}

[1]B.D. Iverson, S.V. Garimella, Recent advances in microscale pumping technologies: a review and evaluation, Microfluid Nanofluid (2008) 5: 145-174.

[2] K.E. Goodson et al. Electro-osmotic microchannel cooling system, U.S. Patent 6,942,018, issued September 13 (2005).

[3] J. Xie, J. Shih, Q. Lin, B. Yang and Y-C. Tai, Surface micro-machined electrostatically actuated micro peristaltic pump, Lab Chip, (2004) 4, 495-501.

[4] T. Brettschneider and C. Dorrer, Microfluidic peristaltic pump, method and pumping system, U.S. Patent Application, 14/075,301 (2013).

[5] H.P. Chou, A.Y. Fu and S. R. Quake, Microfluidic devices and methods of use, U.S. Patent No. 8,992,858. 31 Mar. (2015).

[6] X. Zhang, Z. Chen and Y. Huang, A valve-less microfluidic peristaltic pumping method, Biomicrofluidics, 9, 014118 (2015).

[7] D. Si and Y. Jian, Electro-magneto-hydrodynamic (EMHD) micropump of Jeffrey fluids through two parallel microchannels with corrugated walls, J. Phys. D: Appl. Phys. 48 (2015) 085501 (10pp)

[8] J.C. Burns and T. Parkes, Peristaltic motion, J. Fluid Mechanics, 29 (1967): 731-743.

[9] Y.C. Fung and C.S. Yih, Peristaltic transport, ASME J. Applied Mechanics, 35 (1968): 669-675.

[10] A.H. Shapiro, M.Y. Jaffrin and S.L. Weinberg, Peristaltic pumping with long wavelengths at low Reynolds number, J. Fluid Mechanics, 37 (1969): 799-825.

[11] M.Y. Jaffrin and A. H. Shapiro, Peristaltic pumping, Ann. Rev. Fluid Mechanics, 3 (1971): 13-37.

[12] J.B. Shukla, R. S. Parihar, B. R. P. Rao and S. P. Gupta, Effects of peripheral-layer viscosity on peristaltic transport of a bio-fluid, J. Fluid Mechanics, 97 (1980): 225-237. 
[13] S. Takabatake and K. Ayukawa, Numerical study of two-dimensional peristaltic flows, J. Fluid Mechanics, 122 (1982): 439-465.

[14] C. Pozrikidis, A study of peristaltic flow, J. Fluid Mechanics, 180 (1987): 515-527.

[15] M. Li and J.G. Brasseur, Non-steady peristaltic transport in finite-length tubes, J. Fluid Mechanics, 248 (1993): 129-151.

[16] D. Tripathi and O. Anwar Bég, Peristaltic propulsion of generalized Burgers' fluids through a nonuniform porous medium: A study of chyme dynamics through the diseased intestine, Mathematical Biosciences, 248 (2014): 67-77.

[17] D. Tripathi and O. Anwar Bég, Transient magneto-peristaltic flow of couple stress biofluids: a magneto-hydro-dynamical study on digestive transport phenomena, Mathematical Biosciences, 246.1 (2013): 72-83.

[18] F. Blanchette, The influence of suspended drops on peristaltic pumping, Phys. Fluids 26.6 (2014): 061902.

[19] R. Ellahi, M. Mubashir Bhatti, and K. Vafai, Effects of heat and mass transfer on peristaltic flow in a non-uniform rectangular duct, Int. J. Heat Mass Transfer, 71 (2014): 706-719.

[20] D. Tripathi and O. Anwar Bég, A study on peristaltic flow of nanofluids: Application in drug delivery systems, Int. J. Heat Mass Transfer, 70 (2014): 61-70.

[21] M. Kothandapani and J. Prakash, Effect of radiation and magnetic field on peristaltic transport of nanofluids through a porous space in a tapered asymmetric channel, J. Magnetism Magnetic Materials 378 (2015): 152-163.

[23] N.S. Akbar, M. Raza and R. Ellahi, Influence of induced magnetic field and heat flux with the suspension of carbon nanotubes for the peristaltic flow in a permeable channel, J. Magnetism Magnetic Materials, 381 (2015): 405-415.

[24] O. Anwar Bég, M.M. Rashidi, M.T. Rastegari, Tasveer A. Bég, S.S. Motsa and Amna Halim, DTM-Padé numerical simulation of electrohydrodynamic ion drag medical pumps with electrical Hartmann and electrical Reynolds number effects, Advanced Biotechnology and Bioengineering, 1, 6279 (2013).

[25] S. Chakraborty, Augmentation of peristaltic microflows through electro-osmotic mechanisms, $J$. Phys. D: Appl. Phys., 39 (2006) 5356-5363.

[26] D.A. Saville, Electrokinetic effects with small particles, Ann. Rev. Fluid Mechanics, Vol. 9: 321337 (1977). 
[27] M.A. Vakili, A. Sadeghi, M.H. Saidi and A.A. Mozafari, Electrokinetically driven fluidic transport of power-law fluids in rectangular microchannels, Colloids and Surfaces A:Physicochemical and Engineering Aspects, 414 (2012) 440-456.

[28] S. Chakraborty and D. Paul, Microchannel flow control through a combined electromagnetohydrodynamic transport, J. Physics D: Applied Physics, 39 (2006), 5364.

[29] Dey, D. Chakraborty and S. Chakraborty, Extended Graetz problem for combined electroosmotic and pressure-driven flows in narrow confinements with thick electric double layers, Int. J. Heat Mass Transfer, 55 (2012) 4724-4733.

[29] M. Mohammadi, H. Madadi, J. Casals-Terré, J. Sellarès, Hydrodynamic and direct-current insulator-based dielectrophoresis (H-DC-iDEP) microfluidic blood plasma separation, Anal Bioanal Chem (2015) 407:4733-4744.

[30] Mohsen Sheikholeslami and Rahmat Ellahi, Electrohydrodynamic nanofluid hydrothermal treatment in an enclosure with sinusoidal upper wall, Applied Sciences, 5 (2015) 294-306.

[31] M.F. El-Sayed, M. H. Haroun, D.R. Mostapha, Electrohydrodynamic peristaltic flow of a dielectric Oldroydian viscoelastic fluid in a flexible channel with heat transfer, J. Applied Mechanics and Technical Physics, 55 (2014) 565-577.

[32] P. Goswami, J. Chakraborty, A. Bandopadhyay and S. Chakraborty, Electrokinetically modulated peristaltic transport of power-law fluids, Microvascular Research, 103 (2015). DOI: 10.1016/j.mvr.2015.10.004

[33] H. Lai, A. Folch, Design and dynamic characterization of "Single-stroke" Peristaltic PDMS micropumps, Lab. Chip, 11 (2011) 336-342. .

[34] J.G. Smits, Piezoelectric micropump with 3 valves working peristaltically, Sens. Actuat. A, 21 (1990) 203-206.

[35] T.E. McKnight, C.T. Culbertson, S.C. Jacobson, J.M. Ramsey, Electro-osmotically induced hydraulic pumping with integrated electrodes on microfluidic devices, Anal. Chem. 73 (2001) 40454049.

[36] C.H. Chen, J.G. Santiago, A planar electroosmotic micropump, J. Microelectromech. Syst. 11 (2002) 672-683.

[37] A. Ajdari, Pumping liquids using asymmetric electrode arrays, Phys. Rev. E, 61 (2000) R45-R48.

[38] A.B. Brown, C.G. Smith, A.R. Rennie, Pumping of water with AC electric fields applied to asymmetric pairs of microelectrodes, Phys. Rev. E, 63 (2001) 016305. 
[39] V. Studer, A. Pepin, Y. Chen, A. Ajdari, Fabrication of microfluidic devices for AC electrokinetic fluid pumping, Microelectron. Eng., 61 (2002), 915-920.

[40] Jang, J.S.; Lee, S.S. Theoretical and experimental study of MHD (magnetohydrodynamic) micropump, Sens. Actuat. A, 2000, 80, 84-89.

[41] Lemoff, A.V.; Lee, A.P. An AC magnetohydrodynamic micropump, Sens. Actuat. B, 63 (2000) 178-185.

[42] A.V. Ramana Kumari and G. Radhakrishnamacharya, Effect of slip and magnetic field on peristaltic flow in an inclined channel with wall effects, Int. J. Biomath., 5 (2012), 1250015.

[43] K. Ramesh and M. Devakar, Magnetohydrodynamic peristaltic transport of couple stress fluid through porous medium in an inclined asymmetric channel with heat transfer, J. Magnetism Magnetic Materials, 394 (2015) 335.

[44] R. Ellahi, Rahman S.U.,S. Nadeem and Vafai K., The blood flow of Prandtl fluid through a tapered stenosed arteries in permeable walls with magnetic field, Communications in Theoretical Physics, 63(03) (2015) 353-358.

[45] S. Rashidi, M. Dehghan, R. Ellahic, M. Riaze, and M.T. Jamal-Abad, Study of stream wise transverse magnetic fluid flow with heat transfer around a porous obstacle, J. Magnetism and Magnetic Materials, 378 (2015) 128-137.

[46] Mohsen Sheikholeslami and Rahmat Ellahi Simulation of ferrofluid flow for magnetic drug targeting using Lattice Boltzmann method, Zeitschrift Fur Naturforschung A, 70 (2), (2015) 115-124.

[47]Rahmat Ellahi, Mohsin Hassan, and Ahmad Zeeshan Study on magnetohydrodynamic nanofluid by means of single and multi-walled carbon nanotubes suspended in a salt water solution, IEEE Transactions on Nanotechnology, 14 (4) (2015) 726 - 734.

[48] Noreen Sher Akbar, M. Raza and R. Ellahi, Influence of induced magnetic field and heat flux with the suspension of carbon nanotubes for the peristaltic flow in a permeable channel, J. Magnetism and Magnetic Materials, 381 (2015) 405-415.

[49] M. Sheikholeslami and Rahmat Ellahi, Three dimensional mesoscopic simulation of magnetic field effect on natural convection of nanofluid, Int. J. Heat and Mass Transfer. 89 (2015) 799-808. 